\title{
Controller Design for Continuous-Time Takagi-Sugeno Fuzzy Systems with Fuzzy Lyapunov Functions : LMI Approach
}

\author{
Ho Jun Kim ${ }^{1}$,Young Hoon Joo ${ }^{2, *}$ and Jin Bae Park ${ }^{1}$ \\ 1 Department of Electrical and Electronic Eng., Yonsei Univ., Seoul, 120-749 Korea \\ 2 Department of Control and Robotics Eng., Kunsan National Univ. Kunsan, Chonbuk 573-701, Korea
}

\begin{abstract}
This paper is concerned with stabilization problem of continuous-time Takagi-Sugeno fuzzy systems. To do this, the stabilization problem is investigated based on the new fuzzy Lyapunov functions (NFLFs). The NFLFs depend on not only the fuzzy weighting functions but also their first-time derivatives. The stabilization conditions are derived in terms of linear matrix inequalities (LMIs) which can be solved easily by the Matlab LMI Toolbox. Simulation examples are given to illustrate the effectiveness of this method.
\end{abstract}

Key Words: Fuzzy Lyapunov functions (FLFs); Takagi-Sugeno fuzzy systems; Linear matrix inequalities (LMIs); Stabilization

\section{Introduction}

During the last two decades, Takagi-Sugeno (T-S) fuzzy system is an interesting issue because of its capability to represent nonlinear systems effectively. Nonlinear systems can be represented as an average weighted sum of linear systems by the T-S fuzzy systems. It is very powerful capability because the nonlinear systems can be partially treated by linear control method. The Lyapunov method is main approach to determine stability and solve stabilization problem in the T-S fuzzy systems. In [1], common quadratic Lyapunov function is used for solving stability and stabilization problem. However it has result in very conservative conditions because only one matrix should satisfy all subsystems of the T-S fuzzy systems.

There have been a lot of efforts to resolve this problem. In [2], interactions among all fuzzy subsystems of T-S fuzzy systems are considered. In [3], [4], membership functions are considered in the Lyapunov method. In [5], [6], non-parallel distributed compensation (NPDC), which is the fuzzy controller dose not share the same fuzzy sets with the fuzzy systems, is used, and in [7], [8], the Lyapunov functions are changed for presenting relaxed stability and stabilization conditions. Especially, changing Lyapunov functions is one of the main issues to reduce con-

Manuscript received Jun. 20, 2012; revised Sep. 21, 2012; accepted Sep. 24. 2012.

*Corresponding Author : yhjoo@kunsan.ac.kr

Acknowledgment: This work was supported by the Human Resources Development program(No. 20124010203240) of the Korea Institute of Energy Technology Evaluation and Planning(KETEP) grant funded by the Korea government Ministry of Knowledge Economy.

(c) The Korean Institute of Intelligent Systems. All rights reserved. servativeness. In [7], fuzzy Lyapunov functions (FLFs) are presented at the first time. The FLFs are consist of common Lyapunov functions whose number is the number of fuzzy rules. There have been a lot of efforts to improve the performance of FLFs since those are presented. Especially, continuous-time fuzzy systems (CFSs) are harder to analyze their stability and stabilization than those of the discrete-time fuzzy systems (DFSs) because FLFs of CFSs depend on the time-derivative of the membership functions. In [9], the systematic approach to analyze stability and stabilization of CFSs based on FLFs is proposed by introducing some null terms. In [8], The new fuzzy Lyapunov functions (NFLFs) are proposed which depend on not only the fuzzy weighting functions but also their first-order timederivatives. Although this method is very useful for stability analysis of CFSs, stabilization problem is open issue. The stabilization conditions based on FLFs are derived in terms of bilinear matrix inequalities (BMIs) in the many cases which are harder to be solved than the LMIs. Furthermore, the stabilization conditions based on NFLFs are not yet discussed.

In this paper, stabilization analysis of CFSs based on NFLFs are considered. NFLFs depend on time-derivative of membership functions as well as membership functions. Two assumptions, which are upper bounds of membership functions and those of first time-derivatives, are needed for formula development. Stabilization conditions are derived in terms of LMIs which are easily solved by Matlab LMI Toolbox [10]. Finally, numerical example is given to illustrate the effectiveness of the proposed method.

Notations: $\mathbb{R}^{n}:=n$-dimensional real space. $\mathbb{R}^{m \times n}:=$ Set of all real $m$ by $n$ matrices. $A^{T}:=$ Transpose of matrix 
A. $P \succ 0$ (resp. $P \prec 0$ ) := Positive (resp. negative)definite symmetric matrix. $*:=$ The transposed element in the symmetric position. $h_{i}:=h_{i}(z(t)), x:=x(t), u:=$ $u(t)$.

\section{Preliminaries}

Consider a continuous nonlinear system

$$
\dot{x}=f(x)+g(x) u
$$

where $x \in \mathbb{R}^{n}$ is the state vector, $u \in \mathbb{R}^{m}$ is the input state vector, and $f(x), g(x)$ are smooth nonlinear functions. The $i$-th T-S fuzzy rule for the (1) is represented as

\section{Plant Rule $i$ :}

$$
\begin{aligned}
& \text { If } z_{1}(t) \text { is } F_{1}^{i} \text { and } z_{2}(t) \text { is } F_{2}^{i} \text { and } \ldots z_{p}(t) \text { is } F_{p}^{i} \\
& \text { Then } \dot{x}(t)=A_{i} x(t)+B_{i} u(t),
\end{aligned}
$$

where $i=1,2, \ldots, r$ is the rule number of the fuzzy rules, $F_{j}^{i}$ is the fuzzy sets where $j=1,2, \ldots, p, z_{j}(t)$ is the premise variables, $A_{i} \in \mathbb{R}^{n \times n}$, and $B_{i} \in \mathbb{R}^{n \times m}$ are the $i$-th local system matrices. (2) is described as following equation by using singleton fuzzifier, product inference engine, and center-average defuzzifier.

$$
\dot{x}=\sum_{i=1}^{r} h_{i}\left(A_{i} x+B_{i} u\right)
$$

where $h_{i}$ is the normalized membership function of $i$-th rule and satisfying following properties.

$$
h_{i} \geq 0, \quad \sum_{i=1}^{r} h_{i}=1 \quad(i=1,2, \ldots, r)
$$

The $i$-th rule of the fuzzy controller for the nonlinear system based on parallel distributed compensation (PDC) is described as

\section{Controller Rule $i$ :}

If $z_{1}(t)$ is $F_{1}^{i}$ and $z_{2}(t)$ is $F_{2}^{i}$ and $\ldots z_{p}(t)$ is $F_{p}^{i}$

Then $u=-K_{i} x$,

Using the same fuzzifier, inference engine, and defuzzifier of the plant, (5) is described as:

$$
u=-\sum_{i=1}^{r} h_{i} K_{i} x
$$

and substituting (6) into (3), the final expression is represented as

$$
\dot{x}=\sum_{i=1}^{r} \sum_{j=1}^{r} h_{i} h_{j}\left(A_{i}-B_{i} K_{j}\right) x
$$

In this paper, stabilization analysis is considered based on the NFLF which is described as :

$$
V(x)=\sum_{i=1}^{r} h_{i} x^{T} P_{i} x+\sum_{k=1}^{r} \dot{h_{k}} x^{T} \bar{P}_{k} x
$$

Notice that Lyapunov functions depend on membership function and its first time-derivatives. However, membership function and its time-derivatives are not easy to be handled in terms of LMIs because of their nonlinearity. By this reason, two assumptions, upper bound of first and second time-derivatives, are needed for smooth formula development.

$$
\left|\dot{h}_{k}\right| \leq \phi_{1 k}, \quad\left|\ddot{h}_{l}\right| \leq \phi_{2 l}
$$

where $\phi_{1 k}$ and $\phi_{2 l}$ are positive real number and following properties are derived from the (4)

$$
\sum_{k=1}^{r} \dot{h}_{k}=0, \quad \sum_{l=1}^{r} \ddot{h}_{l}=0
$$

\section{Controller Design}

\subsection{Stability Analysis}

In this section, sufficient stability conditions of the T-S fuzzy system (3) which were proposed in [8] is introduced - Also the NFLF is adopted, and two assumptions (9) are considered. In this section, only the unforced that is, $u=$ 0 , system of (7) is considered, so it is presented as

$$
\dot{x}=\sum_{i=1}^{r} h_{i} A_{i}
$$

Lemma 3.1. [8] T-S fuzzy system (11) is asymptotically stable where (9) is considered, $i, k, l=$ $\{1,2, \ldots, r\}, j>i$ and if there exist symmetric matrices $P_{i}, \bar{P}_{k}, X, Y_{11}, Y_{22}, Z_{11}, Z_{22}$, any matrices $M_{1 i}, M_{2 i}, N_{1 k}, N_{2 k}, L_{1 l}, L_{2 l}, Y_{21}, Z_{21}$, and satisfying following inequalities.

$\bar{P}_{k}+X \succeq 0$,

$-\sum_{k=1}^{r} \phi_{1 k}\left(\bar{P}_{k}+X\right)+P_{i} \succ 0$,

$\Upsilon_{i k}^{1}:=$

$$
\begin{aligned}
& {\left[\begin{array}{cc}
P_{k}-N_{1 k} A_{i}-A_{i}^{T} N_{1 k}^{T}+Y_{11} & * \\
\bar{P}_{k}+N_{1 k}^{T}-N_{2 k} A_{i}+Y_{21} & N_{2 k}+N_{2 k}^{T}+Y_{22}
\end{array}\right] } \\
& \succeq 0, \\
& \Upsilon_{i l}^{2}:=\left[\begin{array}{cc}
\bar{P}_{l}-L_{1 l} A_{i}-A_{i}^{T} L_{1 l}^{T}+Z_{11} & * \\
L_{1 l}^{T}-L_{2 l} A_{i}+Z_{21} & L_{2 l}+L_{2 l}^{T}+Z_{22}
\end{array}\right]
\end{aligned}
$$


Controller Design for Continuous-Time Takagi-Sugeno Fuzzy Systems with Fuzzy Lyapunov Functions : LMI Approach

$$
\begin{gathered}
\succeq 0, \\
\Upsilon_{i j}^{3}+\Upsilon_{j i}^{3} \prec 0
\end{gathered}
$$

where

$$
\begin{aligned}
\Upsilon_{i j}^{3}:= & {\left[\begin{array}{lc}
-M_{1 j} A_{i}-A_{i}^{T} M_{1 j}^{T} & * \\
P_{i}+M_{1 j}^{T}-M_{2 j} A_{i} & M_{2 j}+M_{2 j}^{T}
\end{array}\right] } \\
& +\sum_{k=1}^{r} \phi_{1 k} \Upsilon_{i k}^{1}+\sum_{l=1}^{r} \phi_{2 l} \Upsilon_{i l}^{2}
\end{aligned}
$$

\subsection{Stabilization analysis}

The stability conditions with NFLFs are well discussed in the previous section. However, the stabilization conditions based on the the NFLF is not tackled at all because of its difficulties to be derived in terms of LMIs. In this section, stabilization conditions based on the NFLF are considered. The concept of parallel distributed compensation (PDC) is employed to design a fuzzy controller which shares the same fuzzy rules and fuzzy sets with the plant of the T-S fuzzy system. Two assumptions (9) are considered and following null terms are added.

$2\left(x^{T} M+\dot{x}^{T} \mu_{1} M\right)\left(\dot{x}-\sum_{i=1}^{r} \sum_{j=1}^{r} h_{i} h_{j}\left(A_{i}-B_{i} K_{j}\right)\right)=0$

$2\left(x^{T} \sum_{k=1}^{r} \dot{h_{k}} M+\dot{x}^{T} \sum_{k=1}^{r} \dot{h_{k}} \mu_{2 k} M\right)$

$\times\left(\dot{x}-\sum_{i=1}^{r} \sum_{j=1}^{r} h_{i} h_{j}\left(A_{i}-B_{i} K_{j}\right)\right)=0$

$2\left(x^{T} \sum_{l=1}^{r} \ddot{h}_{l} M+\dot{x}^{T} \sum_{l=1}^{r} \ddot{h}_{l} \mu_{3 l} M\right)$

$\times\left(\dot{x}-\sum_{i=1}^{r} \sum_{j=1}^{r} h_{i} h_{j}\left(A_{i}-B_{i} K_{j}\right)\right)=0$

where $\mu_{1}, \mu_{2 k}$, and $\mu_{3 l}$ are proper scalar values.

Theorem 3.2. T-S fuzzy system (3) is stabilized with the fuzzy controller (6) gains given by $K_{i}=B_{i} R$ where (9) is considered, and if there exist symmetric matrices $P_{i}, \bar{P}_{i}, T_{i}, \bar{T}_{k}, X, \tilde{Y}_{11}, \tilde{Y}_{22}, \tilde{Z}_{11}, \tilde{Z}_{22}, \Theta_{i j}$ any matrices $R, \tilde{Y}_{21}, \tilde{Z}_{21}$, and satisfying following inequalities.

$$
\begin{array}{cc}
\bar{P}_{k}+X \succeq 0, & k=1,2, \ldots, r \\
-\sum_{k=1}^{r} \phi_{1 k}\left(\bar{P}_{k}+X\right)+P_{i} \succ 0, & i, k=1,2, \ldots, r \\
\Upsilon_{i j k}^{1}: & :=\left[\begin{array}{cc}
\Lambda_{i j k}+\tilde{Y}_{11} & * \\
\Xi_{i j k}+\tilde{Y}_{21} & \mu_{2 k}\left(R+R^{T}\right)+\tilde{Y}_{22}
\end{array}\right] \\
\succeq 0, & i, j, k=1,2, \ldots, r \\
\Upsilon_{i j l}^{2}:=\left[\begin{array}{cc}
\prod_{i j l}+\tilde{Z}_{11} & * \\
\Sigma_{i j l}+\tilde{Z}_{21} & \mu_{3 l}\left(R+R^{T}\right)+\tilde{Z}_{22}
\end{array}\right]
\end{array}
$$

$$
\begin{array}{ccc}
\succeq 0, & & i, j, l=1,2, \ldots, r \\
\Upsilon_{i i}^{3}+\Theta_{i i} \prec 0 & & i=1,2, \ldots, r \\
\Upsilon_{i j}^{3}+\Upsilon_{j i}^{3}+2 \Theta_{i j} \prec 0, & 1 \leq i<j \leq r \\
{\left[\begin{array}{cccc}
\Theta_{11} & \Theta_{12} & \cdots & \Theta_{1 r} \\
\Theta_{12} & \Theta_{22} & \cdots & \Theta_{2 r} \\
\vdots & & \ddots & \vdots \\
\Theta_{1 r} & \Theta_{2 r} & \cdots & \Theta_{r r}
\end{array}\right] \succ 0} &
\end{array}
$$

where

$$
\begin{aligned}
& \Upsilon_{i j}^{3}:=\Omega_{i j}+\sum_{k=1}^{r} \phi_{1 k} \Upsilon_{i j k}^{1}+\sum_{l=1}^{r} \phi_{2 l} \Upsilon_{i j l}^{2} \\
& \Lambda_{i j k}:=T_{k}-\left(A_{i} R^{T}-B_{i} S_{j}\right)-\left(A_{i} R^{T}-B_{i} S_{j}\right)^{T} \\
& \Xi_{i j k}:=\bar{T}_{k}+R-\mu_{2 k}\left(A_{i} R^{T}-B_{i} S_{j}\right) \\
& \Pi_{i j l}:=\bar{T}_{l}-\left(A_{i} R^{T}-B_{i} S_{j}\right)-\left(A_{i} R^{T}-B_{i} S_{j}\right)^{T} \\
& \Sigma_{i j l}:=R-\mu_{3 l}\left(A_{i} R^{T}-B_{i} S_{j}\right) \\
& \Omega_{i j}:= \\
& {\left[\begin{array}{cc}
-\left(A_{i} R^{T}-B_{i} S_{j}\right)-\left(A_{i} R^{T}-B_{i} S_{j}\right)^{T} & * \\
T_{i}+R-\mu_{1}\left(A_{i} R^{T}-B_{i} S_{j}\right) & \mu_{1}\left(R+R^{T}\right)
\end{array}\right]}
\end{aligned}
$$

Proof. The (8) should be demonstrated to show that $V(x)$ is a Lyapunov candidate function. Let us consider (9), (21), and (22). First, $V(x)>0$ should be verified.

$$
\begin{aligned}
V(x) & =\sum_{k=1}^{r} \dot{h}_{k} x^{T} \bar{P}_{k} x+\sum_{i=1}^{r} h_{i} x^{T} P_{i} x \\
& =x^{T}\left(\sum_{k=1}^{r} \dot{h}_{k} \bar{P}_{k}+\sum_{i=1}^{r} h_{i} P_{i}+\sum_{k=1}^{r} \dot{h}_{k} X\right) x \\
& =x^{T}\left(\sum_{k=1}^{r} \dot{h}_{k}\left(\bar{P}_{k}+X\right)+\sum_{i=1}^{r} h_{i} P_{i}\right) x \\
& \geq \sum_{i=1}^{r} h_{i} x^{T}\left(-\sum_{k=1}^{r} \phi_{1 k}\left(\bar{P}_{k}+X\right)+P_{i}\right) x \\
& >0 .
\end{aligned}
$$

From now on $\dot{V}(x)<0$ should be demonstrated.

$$
\begin{aligned}
\dot{V}(x)= & \sum_{l=1}^{r} \ddot{h}_{l} x^{T} \bar{P}_{l} x+\sum_{k=1}^{r} \dot{h}_{k} x^{T} P_{k} x \\
& +2 \sum_{k=1}^{r} \dot{h}_{k} \dot{x}^{T} \bar{P}_{k} x+2 \sum_{i=1}^{r} h_{i} \dot{x}^{T} P_{i} x
\end{aligned}
$$

Let us consider null terms (18) - (20).

$$
\begin{aligned}
\dot{V}(x)= & \sum_{l=1}^{r} \ddot{h}_{l} x^{T} \bar{P}_{l} x+\sum_{k=1}^{r} \dot{h}_{k} x^{T} P_{k} x \\
& +2 \sum_{k=1}^{r} \dot{h}_{k} \dot{x}^{T} \bar{P}_{k} x+2 \sum_{i=1}^{r} h_{i} \dot{x}^{T} P_{i} x
\end{aligned}
$$


International Journal of Fuzzy Logic and Intelligent Systems, vol.12, no. 3, September 2012

$$
\begin{aligned}
& +2\left(x^{T} M+\dot{x}^{T} \mu_{1} M\right) \\
& \times\left(\dot{x}-\sum_{i=1}^{r} \sum_{j=1}^{r} h_{i} h_{j}\left(A_{i}-B_{i} K_{j}\right)\right) \\
& +2\left(x^{T} \sum_{k=1}^{r} \dot{h_{k}} M+\dot{x}^{T} \sum_{k=1}^{r} \dot{h_{k}} \mu_{2 k} M\right) \\
& \times\left(\dot{x}-\sum_{i=1}^{r} \sum_{j=1}^{r} h_{i} h_{j}\left(A_{i}-B_{i} K_{j}\right)\right) \\
& +2\left(x^{T} \sum_{l=1}^{r} \ddot{h_{l}} M+\dot{x}^{T} \sum_{l=1}^{r} \ddot{h_{l}} \mu_{3 l} M\right) \\
& \times\left(\dot{x}-\sum_{i=1}^{r} \sum_{j=1}^{r} h_{i} h_{j}\left(A_{i}-B_{i} K_{j}\right)\right)
\end{aligned}
$$

Take a congruence transformation to represent in terms of LMI and consider (9).

$$
\begin{aligned}
& \sum_{i=1}^{r} \sum_{j=1}^{r} h_{i} h_{j}\left[\begin{array}{l}
x \\
\dot{x}
\end{array}\right]^{T} \Omega_{i j}\left[\begin{array}{l}
x \\
\dot{x}
\end{array}\right] \\
& +\sum_{i=1}^{r} \sum_{j=1}^{r} \sum_{k=1}^{r} h_{i} h_{j} \dot{h}_{k} \\
& {\left[\begin{array}{c}
x \\
\dot{x}
\end{array}\right]^{T}\left[\begin{array}{cc}
\Lambda_{i j k}+\tilde{Y}_{11} & * \\
\Xi_{i j k}+\tilde{Y}_{21} & \mu_{2 k}\left(R+R^{T}\right)+\tilde{Y}_{22}
\end{array}\right]\left[\begin{array}{c}
x \\
\dot{x}
\end{array}\right]} \\
& +\sum_{i=1}^{r} \sum_{j=1}^{r} \sum_{l=1}^{r} h_{i} h_{j} \ddot{h}_{l} \\
& {\left[\begin{array}{c}
x \\
\dot{x}
\end{array}\right]^{T}\left[\begin{array}{cc}
\Pi_{i j l}+\tilde{Z}_{11} & * \\
\Sigma_{i j l}+\tilde{Z}_{21} & \mu_{3 l}\left(R+R^{T}\right)+\tilde{Z}_{22}
\end{array}\right]\left[\begin{array}{c}
x \\
\dot{x}
\end{array}\right]} \\
& =\sum_{i=1}^{r} \sum_{j=1}^{r} h_{i} h_{j} \\
& {\left[\begin{array}{l}
x \\
\dot{x}
\end{array}\right]^{T}\left(\Omega_{i j}+\sum_{k=1}^{r} \dot{h}_{k} \Upsilon_{i j k}^{1}+\sum_{l=1}^{r} \ddot{h}_{l} \Upsilon_{i j l}^{2}\right)\left[\begin{array}{l}
x \\
\dot{x}
\end{array}\right]} \\
& \leq \sum_{i=1}^{r} \sum_{j=1}^{r} h_{i} h_{j} \\
& {\left[\begin{array}{l}
x \\
\dot{x}
\end{array}\right]^{T}\left(\Omega_{i j}+\sum_{k=1}^{r} \phi_{1 k} \Upsilon_{i j k}^{1}+\sum_{l=1}^{r} \phi_{2 l} \Upsilon_{i j l}^{2}\right)\left[\begin{array}{l}
x \\
\dot{x}
\end{array}\right]} \\
& =\left[\begin{array}{c}
x \\
\dot{x}
\end{array}\right]^{T}\left(\sum_{i=1}^{r} h_{i}^{2} \Upsilon_{i i}^{3}+\sum_{i=1}^{r} \sum_{j>i}^{r}\left(\Upsilon_{i j}^{3}+\Upsilon_{j i}^{3}\right)\right)\left[\begin{array}{l}
x \\
\dot{x}
\end{array}\right] \\
& \leq\left[\begin{array}{c}
x \\
\dot{x}
\end{array}\right]^{T}-\left(\sum_{i=1}^{r} h_{i}^{2} \Theta_{i i}+\sum_{i=1}^{r} \sum_{j>i}^{r} 2 \Theta_{i j}\right)\left[\begin{array}{l}
x \\
\dot{x}
\end{array}\right] \\
& =-\left[\begin{array}{c}
h_{1} z \\
h_{2} z \\
\vdots \\
h_{r} z
\end{array}\right]^{T}\left[\begin{array}{cccc}
\Theta_{11} & \Theta_{12} & \cdots & \Theta_{1 r} \\
\Theta_{12} & \Theta_{22} & \cdots & \Theta_{2 r} \\
\vdots & & \ddots & \vdots \\
\Theta_{1 r} & \Theta_{2 r} & \cdots & \Theta_{r r}
\end{array}\right]\left[\begin{array}{c}
h_{1} z \\
h_{2} z \\
\vdots \\
h_{r} z
\end{array}\right] \\
& <0 \\
& \text { where } z^{T}=\left[\begin{array}{ll}
x^{T} & \dot{x}^{T}
\end{array}\right]
\end{aligned}
$$

Therefore, if (27) is satisfied, $\dot{V}$ is negative definite and the equilibrium point of the fuzzy system is stabilized with the fuzzy controller (6).

Remark 3.3. The contribution of this paper is that the stabilization conditions are derived in terms of LMIs based on the new fuzzy Lyapunov function.

Remark 3.4. Theorem 1 can induce conservative results according to changes in the value of the parameters $\mu_{1}, \mu_{2 k}$, and $\mu_{3 l}$. There are no ways to choose these scalar vector systematically but just do trial and error for set these parameters. The main factor of the conservativeness may 


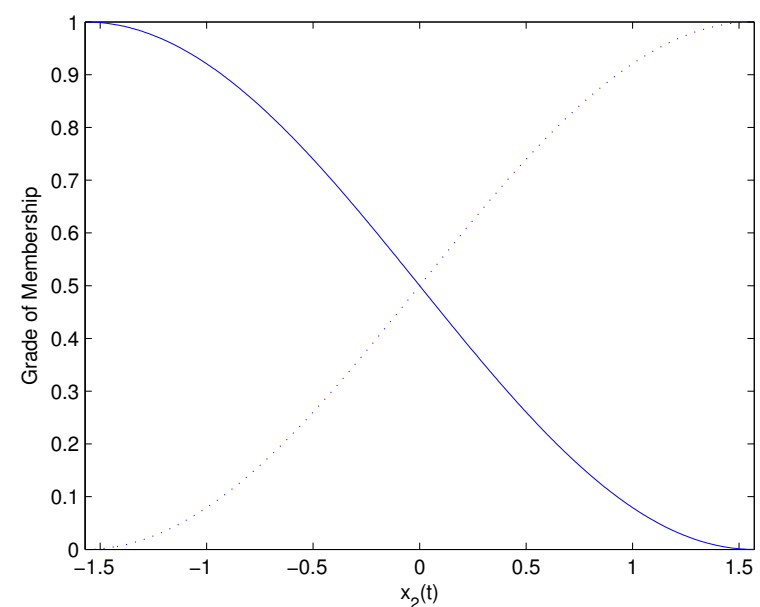

Figure 1: Membership functions of the fuzzy model. (dotted line : $h_{1}$, dashed line $: h_{2}$ )

be the common matrix $M$ between the (18) - (20). Therefore, it is an open issue to remove the common matrix for solving the problem.

\section{Computer Simulations}

To show the effectiveness of the proposed method, numerical example is given and performed with the Matlab LMI Toolbox. Consider the fuzzy system (3) with

$$
\begin{aligned}
& A_{1}=\left[\begin{array}{cc}
2 & -10 \\
1 & 0
\end{array}\right], A_{2}=\left[\begin{array}{cc}
16 & -10 \\
1 & 0.65
\end{array}\right], \\
& B_{1}=\left[\begin{array}{l}
1 \\
0
\end{array}\right], B_{2}=\left[\begin{array}{l}
3 \\
0
\end{array}\right]
\end{aligned}
$$

where $\phi_{1 k}=\phi_{2 k}=0.8, \mu_{1}=\mu_{2 k}=\mu_{3 l}=1$, and $x(0)=\left[\begin{array}{ll}1 & 1\end{array}\right]^{T}$.

The membership functions are

$$
h_{1}=\frac{1+\sin x_{2}(t)}{2}, h_{2}=\frac{1-\sin x_{2}(t)}{2}
$$

, and $x_{2}(t) \in[-\pi / 2 \pi / 2]$. The Fig. 1 shows the membership functions of the fuzzy model. The feedback gains are obtained in the Theorem 1 as

$$
K_{1}=\left[\begin{array}{ll}
6.9924 & 0.8427
\end{array}\right], \quad K_{2}=\left[\begin{array}{ll}
7.0212 & 1.0479
\end{array}\right]
$$

The Fig. 2 and Fig. 3 show the state trajectory of the each state variable $x_{1}$ and $x_{2}$ with controller feedback gains (28). All states of the system go to zero, and the fuzzy system (3) is stabilized with the fuzzy controller (6).

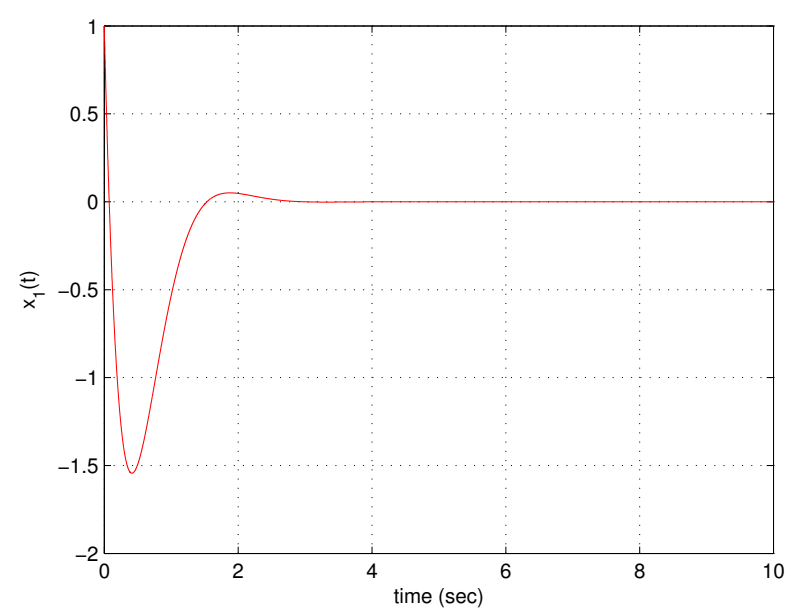

Figure 2: The system state response of $x_{1}(t)$ with the fuzzy controller

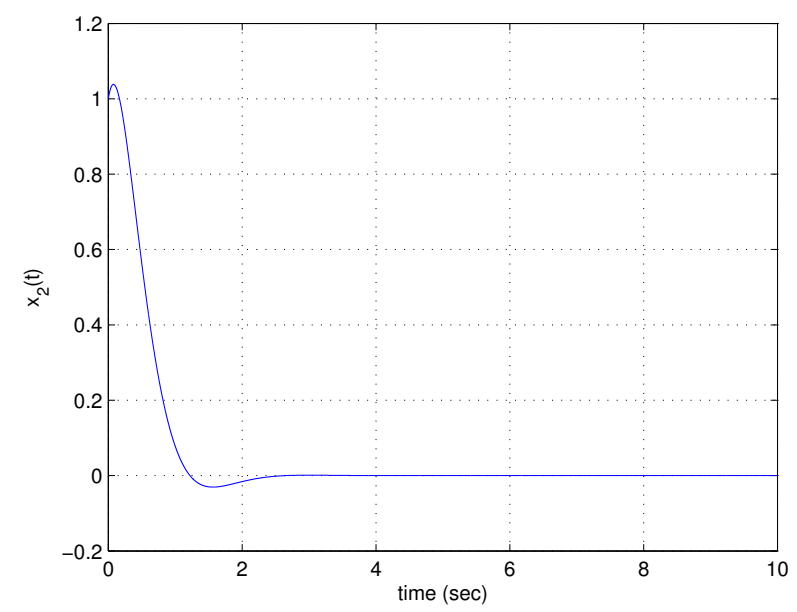

Figure 3: The system state response of $x_{2}(t)$ with the fuzzy controller

\section{Conclusion}

In this paper, the stabilization conditions with NFLFs are given. The NFLFs depend on not only the fuzzy weighting functions but also their first-time derivatives. The stabilization conditions are derived in terms of LMIs and easily solved by the LMI Toolbox. There are open issues to solve the common matrix problem which induce the conservativeness. Simulation example is given to show the effectiveness of this method.

\section{References}

[1] K. Tanaka, and M. Segeno, "Stability analysis and de- 
sign of fuzzy control of nonlinear systems: Stability and the design issues," Fuzzy Sets Syst., vol. 45, np. 2, pp. 1697-1700, 1992.

[2] E. T. Kim, and H. J Lee, "New approaches to relaxed quadratic stability conditions of fuzzy control systems," IEEE Trans. Fuzzy Syst., vol. 8, no. 5, pp. 523-534, 2000.

[3] A. Sala, and C. Arino, "Relaxed stability and performance LMI conditions for Takagi-Sugeno fuzzy systems with polynomial constraints on membership function shapes," IEEE Trans. Fuzzy Syst., vol. 16, no. 5, pp.1328-1336, 2008.

[4] H. K. Lam, "LMI-based stability analysis for fuzzymodel-based control systems using artificial T-S fuzzy model," IEEE Trans. Fuzzy Syst., vol. 19, no. 3, pp. 505-513, 2011.

[5] H. K. Lam and M. Narimani, "Stability Analysis and Performance Design for Fuzzy-Model-Based Control System Under Imperfect Premise Matching," IEEE Trans. Fuzzy Syst., vol. 17, no. 4, pp. 949-961, 2009.

[6] T. M. Guerra, and L. Vermeiren, "LMI-based relaxed nonquadratic stabilization conditions for nonlinear systems in the Takagi-Sugeno's form," Automatica, vol. 40, no. 5 pp. 823-829, 2004.

[7] K. Tanaka, T. Hori, and H. O. Wang, "A multiple lyapunov function approach to stabilization of fuzzy control systems," IEEE Trans. Fuzzy Syst., vol. 11, no. 4, pp. 582-589, 2003.
[8] D. H. Lee, J. B. Park, and Y. H. Joo, "A new fuzzy lyapunov function for relaxed stability condition of continuous-time Takagi-Sugeno fuzzy systems," IEEE Trans. Fuzzy Syst., vol. 19, no. 4, pp. 785-791, 2011.

[9] L. A. Mozelli, R. M. Palhares, and G. S. C. Avellar, "A systematic approach to improve multiple Lyapunov function stability and stabilization conditions for fuzzy systems," Information Science, vol. 179, no. 8, pp. 1149-1162, 2009.

[10] Gahinet, P., Nemirovski, A., Laub., A. J., and Chilali, M. (1995), "LMI control toolbox. MathWorks,", Natick, Massachusetts.

\section{Ho Jun Kim}

Student of the Yonsei University

Research Area: Fuzzy Control

E-mail : khj08121@yonsei.ac.kr

\section{Young Hoon Joo}

Professor of the Kunsan National University

Research Area: Fuzzy, Nonlinear Systems

E-mail : yhjoo@kunsan.ac.kr

\section{Jin Bae Park}

Professor of the Yonsei University

Research Area: Nonlinear System, Filter, Fuzzy

E-mail : jbpark@yonsei.ac.kr 